\title{
ANTAGONISTAS DE RECEPTORES DE PROGESTERONA (PRA) Y MODULADORES SELECTIVOS DE RECEPTORES DE PROGESTERONA (SPRM): APLICACIONES EN GINECOLOGÍA Y OBSTETRICIA
}

\section{Progesterone receptor antagonists (PRAs) and progesterone receptor selective modulators (SPRMs): applications in gynaecology and obstetrics}

Diana Marcela Suárez-Calderón, M.D. *, Ivonne Díaz-Yamal, M.D. **

Recibido: diciembre 13/06 - Aceptado: marzo 7/08

\section{RESUMEN}

Dos décadas de estudio bioquímico, farmacológico y clínico han permitido la síntesis de antagonistas del receptor de la progesterona (PRA) y moduladores selectivos de receptores de progesterona (SPRM). El receptor de progesterona (PR) comparte la estructura de los receptores esteroideos que al ser estimulados sufren un cambio conformacional y subsecuentemente activan la transcripción genética en un tejido específico. Dicho proceso depende, particularmente en el PR, del equilibrio existente entre coactivadores y correpresores que ya han sido identificados. El mifepristone ha sido catalogado como PRA, mientras que el asoprisnil es el clásico SPRM agonista-antagonista. Múltiples estudios experimentales y clínicos han evidenciado la utilidad de los PRA y los SPRM en escenarios clínicos tan comunes como la miomatosis uterina, la endometriosis, la terminación del embarazo y la anticoncepción. Sin embargo, aún no existe la molécula ideal y faltan estudios que respalden la

\footnotetext{
* Ginecoobstetra Hospital Militar Central. Bogotá, Colombia. Correo electrónico: lunaroja.diana@gmail.com

** Coordinadora de la Unidad de Infertilidad Hospital Militar Central Bogotá, Colombia.
}

seguridad y eficacia de mifepristone y asoprisnil a largo plazo. El objetivo del presente documento es revisar la farmacodinamia y usos clínicos de estos medicamentos en ginecología y obstetricia.

Palabras clave: receptores de progesterona. antiprogestina, mifepristone, asoprisnil, aborto, miomatosis, endometriosis, anticoncepción de emergencia.

\section{SUMMARY}

Biochemical, pharmacological and clinical studies have led to synthesizing progesterone receptor antagonists (PRAs) and selective progesterone receptor modulators (SPRMs) during the last two decades. The progesterone receptor (PR) shares its structure with that of other steroid receptors which undergo a conformational change after being stimulated and consequently activate genetic transcription in specific tissues. This process depends (particularly in the PA) on the balance between previously identified co-activators and co-repressors. Mifepristone has been catalogued as being PRA whilst asoprisnil is the classical SPRM agonist-antagonist. Multiple experimental and clinical trials have shown the real usefulness of PRAs 
and SPRMs in everyday clinical scenarios such as uterine myomatosis, endometriosis, termination of pregnancy and contraception. Nevertheless, there is no ideal SPRM and studies supporting the safety and efficacy of mifepristone and asoprisnil are still lacking. This article was aimed at reviewing the pharmacodynamic and clinic uses of these medications in obstetrics and gynaecology.

Key words: progesterone receptors, antiprogestin, mifepristone, asoprisnil, abortion, myoma, endometriosis, emergency contraception.

\section{INTRODUCCIÓN}

Extensos análisis bioquímicos, farmacológicos y clínicos han permitido la síntesis de antagonistas del receptor de progesterona y moduladores selectivos de receptores de progesterona. El receptor de progesterona comparte la estructura de los receptores esteroideos que al ser estimulados sufren un cambio conformacional, activando la transcripción genética en un tejido específico. Este proceso depende del equilibrio entre coactivadores y correpresores.

El mifepristone ha sido catalogado como PRA, mientras que el asoprisnil es el clásico SPRM agonista-antagonista. Múltiples estudios han resaltado la utilidad de los PRA y los SPRM en escenarios clínicos como la miomatosis uterina, la endometriosis, la terminación del embarazo y la anticoncepción. No obstante, aún no existe la molécula ideal y no se cuenta con estudios que respalden la eficacia y seguridad de estos agentes a largo plazo.

\section{DEFINICIÓN}

Los moduladores selectivos de receptores de progesterona (SPRM), también conocidos como mesoprogestinas o agonistas-antagonistas parciales; y los antagonistas de receptores de progesterona (PRA), son moléculas de origen hormonal o no hormonal capaces de generar respuestas agonistas/antagonistas de progesterona sobre tejidos específicos que han sido catalogadas mediante el test de McPhail, que determina la actividad progestacional de un compuesto in vivo, sobre el útero de conejos in- maduros estimulados inicialmente con estradiol y luego con el compuesto de estudio. Las biopsias son clasificadas de 0 a 4, siendo 4 la máxima respuesta posovulatoria. ${ }^{1}$

La mayoría de estos compuestos actúan mediante un balance en la expresión de coactivadores como SRC (steroid receptor coactivador) o CBP (CREB binding protein) y correpresores como NCoR (nuclear correpresor) y SMRT (silencing mediador of retinoid and thyroid hormona), lo cual determina su actividad agonistaantagonista sobre una célula específica. ${ }^{2}$ Figura 1

\section{Receptores de progesterona (PR)}

El receptor de progesterona (PR), al igual que los receptores de glucocorticoides, mineralocorticoides, andrógenos, estrógenos y vitamina $\mathrm{D}$, es un ligando natural de la superfamilia de los receptores nucleares para el control de funciones biológicas a través de la regulación de genes específicos involucrados en el desarrollo embriológico, reproducción, crecimiento, diferenciación tisular y homeostasis hormonodependiente. ${ }^{3}$

El PR presenta dos isoformas, PR-A y PR-B, dependiendo de la acción de dos promotores diferentes (hPR-A y hPR-B) que determinan su expresión celular como homodímeros (AA-BB) o heterodímeros (AB).

Como la relación hPR-A/hPR-B varía en diferentes situaciones fisiológicas y patológicas, la respuesta está determinada por la concentración celular de la isoforma específica. Por ejemplo, en mama y endometrio el PR-A inhibe al PR-B y a los receptores de estrógeno (ER) reduciendo la proliferación epitelial. ${ }^{4}$

La porción aminoterminal del PR contiene dominios con función activadora (AF1 y AF3) que reclutan proteínas coactivadoras facilitando la remodelación cromatínica y la formación de complejos iniciadores de la transcripción en el promotor específico. En el extremo carboxiterminal del dominio de unión al ADN (DBD) se ubica el ligando de unión al dominio (LBD) que es la región más constante del receptor. El DBD consta de dos proyecciones de zinc 


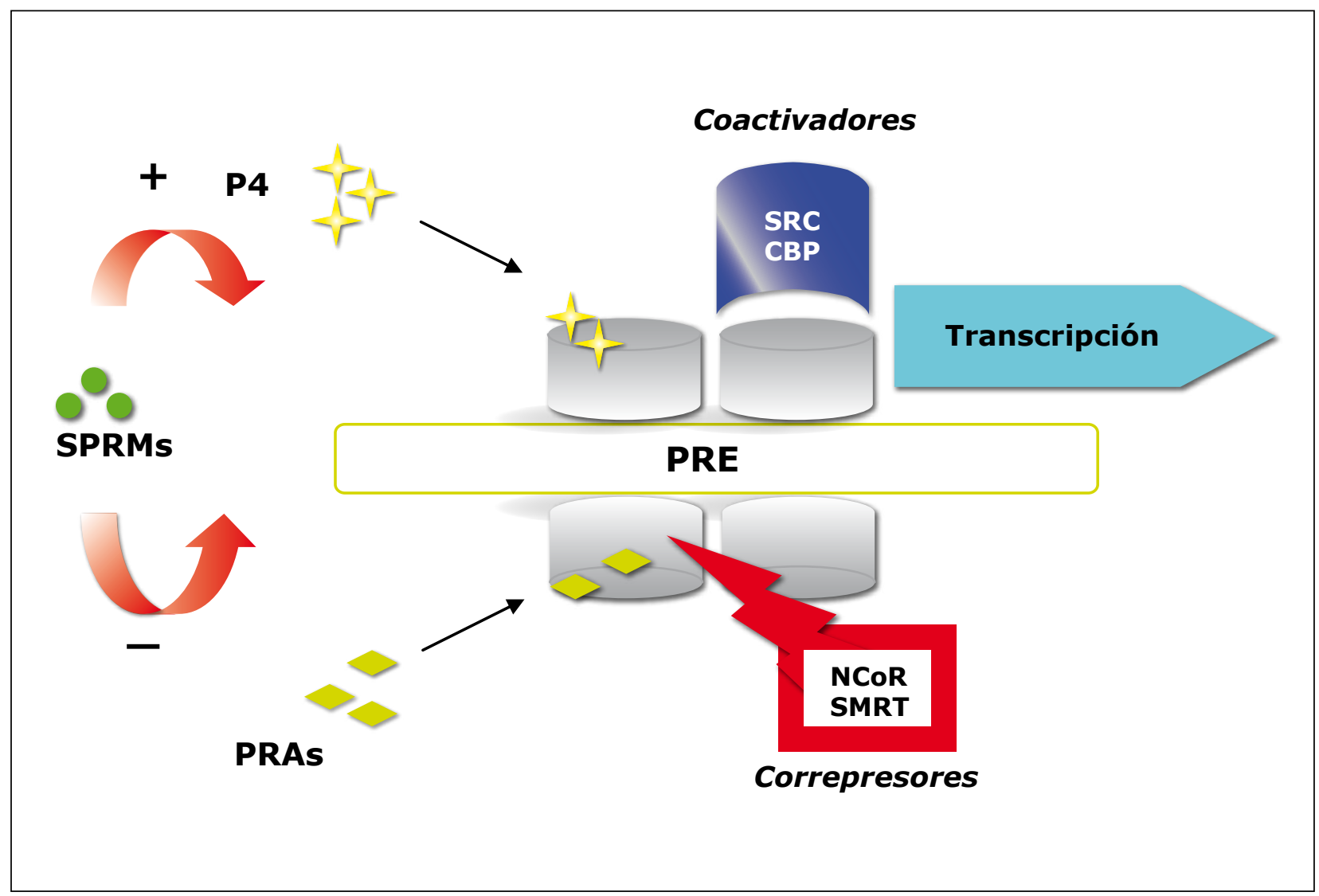

Figura 1. Mecanismo de acción de los PRA y los SPRM.

asimétricas y dos alfa hélices perpendiculares una a la otra que facilitan la interacción con elementos de respuesta a la progesterona (PRE), presentes en los genes iniciadores (ATP1A1, CLDN8, MAN1A1, SEC14L2).

\section{Antagonistas de receptores de progesterona (PRA)}

En 1981 Philibert, Deraedt y Teutsch descubrieron un nuevo antagonista de receptores de glucocorticoides (GR) con moderada actividad antiprogestina, el RU486 conocido con el nombre genérico de mifepristone.

El mifepristone es un antagonista de los receptores de progesterona (PRA), derivado de la noretindrona, con un grupo fenil (4-dimetilamino) en la posición 11 beta responsable de la actividad antagonista y una cadena 1-propinil en la posición 17 alfa, de alta afinidad por el PR que actúa mediante bloqueo de coactivadores y reclutamiento de correpresores. Posteriormente se sintetizó el onapristone (ZK98299) y el ZK137316 que tienen una estructura similar al mifepristone. ${ }^{5}$ Este agente tiene una vida media de 20 a 50 horas. Se liga fuertemente a la albúmina y ligeramente a la SHBG (sexual hormone binding globulin). Se metaboliza en el hígado (citocromo P450 3A4) a J912 que ha demostrado ser un SPRM.

Su uso prolongado con dosis de $200 \mathrm{mg}$ /día puede generar fatiga, náuseas, anorexia, vómito, pérdida de peso, rash cutáneo, amenorrea, fragilidad del pelo, oleadas de calor, disminución de la libido y ginecomastia en hombres, por bloqueo de baja afinidad de receptores androgénicos (AR). Su efecto antiglucocorticoide puede generar hipotiroidismo bioquímico al inhibir la recaptación de yodo inducido por la hidrocortisona y la TSH. Puede haber aumento transitorio de enzimas hepáticas, hipocalemia e hipoadrenalismo. ${ }^{6}$

La hiperplasia endometrial se presenta en el $20 \%$ de las pacientes con mifepristone 5 a 10 $\mathrm{mg}$ /día durante seis meses y es reversible con la suspensión del tratamiento. Bajas dosis (2 a $5 \mathrm{mg}$ / día) se asociaron a cambios quísticos y estromales 
inactivos (sin hiperplasia ni atipia). Este efecto estrogénico del mifepristone se debe a que actúa como un potente antagonista de ER-beta, que es antiproliferativo, permitiendo que se active ER-alfa sin oposición. También un aumento de la relación $\mathrm{PR}-\mathrm{B} / \mathrm{PR}-\mathrm{A}$ permite que aumente la respuesta uterina al estradiol.

El PRA 910 es un derivado no esteroideo de la 6-aril benzoxazinona y ejerce un efecto antagonista de PR comparable con mifepristone. ${ }^{8}$ El CDB2914 es un derivado de la 19-norprogesterona que actúa como antagonista tisular de la progesterona al igual que el mifepristone, pero a diferencia de él, tiene baja actividad antiglucocorticoide. ${ }^{9}$

\section{Moduladores selectivos de receptores de progesterona (SPRM)} El asoprisnil (J867) es un SPRM "J compuesto" con un grupo sustituto de oximas hidrofóbicas y tiene efectos tanto agonistas como antagonistas del PR. El sufijo "isnil" distingue los SPRM del "pristone" de los PRA.

Asoprisnil tiene alta afinidad por los PR uterinos y baja por los GR y los AR. No se liga a ER ni a receptores mineralocorticoides y tiene menos actividad antagonista PR que el mifepristone sobre el endometrio. A nivel periférico, asoprisnil inhibe la actividad de la aromatasa (CYP 19) medida por la conversión de androstenediona a estrona. Se metaboliza al antagonista J912 el cual alcanza concentraciones cinco veces mayores que las del asoprisnil, por lo tanto, el efecto in vivo depende del balance entre asoprisnil y J912.

Las biopsias de endometrio evidencian diferenciación asincrónica del epitelio y el estroma endometrial, siendo el adelgazamiento de las paredes arteriolares el verdadero origen de la supresión menstrual. La amenorrea inducida por el uso de asoprisnil durante 12 semanas es del $50 \%$ con $5 \mathrm{mg} /$ día, $71 \%$ con $10 \mathrm{mg}$ /día y 93\% con $25 \mathrm{mg} /$ día. Los principales efectos secundarios son cefalea, dolor abdominal, náusea, vértigo y metrorragia. ${ }^{10}$
Otros SPRM como dex-mesilato, dex-oxetanona, J912, J956, J1042, LG 120920, ORG33628, ORG 31710 han sido menos estudiados, por lo cual existen pocas publicaciones.

El SPRM ideal deberá tener el potencial para proveer efecto antiproliferativo en el endometrio y seno, efecto protector estrogénico en ovario, hueso y sistema cardiovascular; y efecto neutral sobre el sistema nervioso central.

\section{Aplicaciones clínicas en ginecología y obstetricia}

Son múltiples y prometedoras las aplicaciones clínicas de los SPRM. Sin embargo, en la actualidad, su uso rutinario solo ha sido aprobado para el mifepristone en la terminación del embarazo en primer y segundo trimestre.

\section{Termina ción del embarazo en primer}

trimestre

El esquema más estudiado consiste en 200 mg vía oral dosis única de mifepristone, seguidos 24 a 36 horas después por misoprostol $800 \mu \mathrm{g}$ (oral o vaginal) o gemeprost (pesario vaginal) y un periodo de observación de 4 a 6 horas para evaluar la respuesta. Las tasas de éxito en embarazos menores o iguales a 63 días alcanzan rangos entre el 93,2\% y el 99,6\%. Dos estudios controlados aleatorizados generan controversia acerca de la efectividad de la administración simultánea de $200 \mathrm{mg}$ de mifepristone y $800 \mu \mathrm{g}$ de misoprostol, el mayor de ellos incluyó 1.128 mujeres sin encontrar diferencia significativa en la tasa de aborto completo versus el esquema diferido. ${ }^{11,12}$

Para la terminación del embarazo entre las 9 y las 13 semanas se ha probado un esquema con mifepristone $200 \mathrm{mg}$, seguido de cinco dosis de misoprostol, la primera de $800 \mu \mathrm{g}$ seguida de cuatro dosis adicionales de $400 \mu \mathrm{g}$ vía oral o vaginal con intervalos de tres horas; con tasas de efectividad del 90 al 95\% que declina conforme avanza la edad gestacional. ${ }^{13,14}$

Recientes publicaciones acerca del choque séptico por Clostridium sordellii inducido por mifepristone 
requieren ser soportadas por la evidencia, ya que no se ha logrado establecer una verdadera relación causa-efecto. ${ }^{15}$

\section{Termina ción del embarazo en el segundo trimestre}

La administración de mifepristone, previa a los prostaglandínicos, reduce el intervalo de inducción del parto con menos requerimiento analgésico y mejoría de las tasas de éxito. Como reduce las dosis requeridas de prostaglandinas, disminuye también los efectos secundarios. ${ }^{16}$

En casos de óbito fetal se ha usado mifepristone a dosis de $200 \mathrm{mg}$, dos a tres veces al día, por dos días, o monodosis de $200 \mathrm{mg}$ seguido de misoprostol. Si el trabajo de parto no inicia después de 72 horas se debe recurrir a otro método. ${ }^{17}$

\section{Inducción del parto}

Una revisión sistemática que incluyó siete ensayos clínicos controlados demuestra que el mifepristone favorece la maduración cervical, aumenta la probabilidad de parto a las 48 horas (RR 2,82; IC 95\% 1,82 - 4,36) y reduce la probabilidad de cesárea (RR 0,7; IC 95\% 0,53 - 0,95). ${ }^{18}$ Sin embargo, su uso no ha sido aprobado ya que incrementa la incidencia de polisistolia, ruptura uterina, bradicardia e hiperaldosteronismo fetal. ${ }^{19}$

\section{Anticoncepción}

El uso de mifepristone solo de 2 a $5 \mathrm{mg}$ semanales bloquea la ovulación pero se acompaña de irregularidad menstrual y oleadas de calor que genera pobre adherencia al tratamiento.

Se han utilizado regímenes combinados de hasta $50 \mathrm{mg}$ al día de mifepristone durante 14 a 21 días, seguido de progestinas del día 16 al 28 con buenos resultados y pocos efectos secundarios. A la acción anticonceptiva, explicable no solo por la inhibición de la ovulación sino también por alteraciones del moco cervical, se suma la disminución de la cantidad de sangrado menstrual y la regularización de los ciclos. También una dosis única de 200 mg de mifepristone dos días después del pico de hormona luteinizante $(\mathrm{LH})$ previenen el embarazo en un 97\%, pero este momento es difícil de identificar en la práctica clínica. ${ }^{20}$

No ha sido encontrado el SPRM ideal para uso combinado con progestinas que no comprometa la inhibición de la ovulación como resultado de la competitividad entre la progestina y el antiprogestágeno por PR en el hipotálamo hipofisiario. ${ }^{21}$

Para la anticoncepción de emergencia el mifepristone tiene dos ventajas sobre los métodos tradicionales y es que además de tener menos efectos secundarios, mantiene su efectividad hasta 120 horas después del coito sin protección. Aun así, su eficacia es inversamente proporcional al tiempo poscoito con porcentajes de falla que van desde el $0,9 \%$ en las primeras 24 horas hasta 2,9\% entre las 96 y las 120 horas. Su uso repetido está contraindicado en un mismo ciclo menstrual y la dosis mínima recomendada es de $25 \mathrm{mg}^{22}$

\section{Fertilización in vitro}

Mifepristone estimula el pico de LH y retarda la maduración endometrial ampliando la ventana de implantación. Aún se requieren más estudios para su aplicación. ${ }^{23}$

\section{Endometriosis}

El mifepristone promueve la apoptosis por sobreexpresión de bax (gen promotor de la apoptosis) y regulación en menos de bcl-2 (gen antiapoptosis) mediante la activación del factor de transcripción NF-KB ligado a promotores de bax y bcl-2. ${ }^{24}$

Mifepristone $100 \mathrm{mg}$ al día por tres meses o 5 a $50 \mathrm{mg}$ al día por seis meses ha demostrado mejoría sintomática y regresión de lesiones visibles hasta en un 55\%, sin disminución de la densidad mineral ósea, ofreciendo ventajas frente a los análogos de la GnRH. ${ }^{25}$

Asoprisnil suprime la producción endometrial de prostaglandinas, específicamente prostaglandina F2 alfa, con reducción en la expresión de COX-2 en cerdos de Guinea. ${ }^{26}$ 
Dos ensayos clínicos aleatorizados con asoprisnil demostraron que $5 \mathrm{mg}$ al día es la mínima dosis efectiva para el control del dolor en pacientes con endometriosis. Ambos estudios mostraron un buen perfil de seguridad y tolerabilidad con este medicamento a corto plazo. ${ }^{27} \mathrm{El}$ asoprisnil ha resultado seguro en mujeres premenopáusicas sanas. ${ }^{28}$

\section{Miomatosis}

Se ha demostrado un aumento en las concentraciones de PR-A y PR-B en leiomiomas, comparadas con las del miometrio adyacente. Los PRA producen atrofia de las arterias espirales que reduce la perfusión endometrial. También bloquean factores de crecimiento de progesterona dependientes como el de queratinocitos. La angiogénesis se inhibe por una vía supresora del VEGF (vascular endotelial growth factor) y bloqueo del ciclo celular en la interfase G2mitosis. ${ }^{29}$

Mifepristone en dosis de 5 a $50 \mathrm{mg}$ al día por 3 a 6 meses, disminuye el volumen de los leiomiomas de un $26 \%$ a un $74 \%$ con tasas de amenorrea que van del 63 al 100\% asociadas a una mejoría del dolor pélvico hasta en un 75\%. Todas las pacientes retornaron a ciclos menstruales regulares 2 a 6 semanas después de suspendido su uso. ${ }^{30} \mathrm{Al}$ comparar la eficacia de mifepristone versus análogos de la GnRH, en reducción del volumen del mioma, se obtuvieron resultados similares (74\% vs. 67\%, respectivamente). ${ }^{31}$

El asoprisnil en dosis de 10 a $25 \mathrm{mg}$ al día por 12 semanas genera una supresión dosis dependiente, tanto de la duración como de la intensidad del sangrado uterino, sin inducir sangrados por atrofia; incrementa las concentraciones de hemoglobina y reduce el volumen uterino y el dolor pélvico sin el riesgo de hiperplasia endometrial que se presenta con mifepristone. ${ }^{32}$

\section{Tumores con receptores esteroideos}

Estudios preclínicos han demostrado la eficacia del mifepristone para inhibir el crecimiento del cáncer epitelial de ovario, a través de la activación de inhi- bidores de la ciclina dependiente de quinasa (cdk) y la reducida trascripción de factores que expresan cdk2 y ciclinas A y E. ${ }^{33}$

Una paciente con leiomiosarcoma recurrente de bajo grado obtuvo una dramática respuesta al tratamiento con mifepristone $50 \mathrm{mg}$ /día, al cabo de tres años. En otras dos pacientes, con recurrencias de alto grado, la enfermedad progresó en los meses siguientes. ${ }^{34}$

\section{REFERENCIAS}

1. McPhail MK. The assay of progestins. J Physiol 1934;83:145-56.

2. Spitz I. Progesterone antagonists and progesterone receptor modulators: an overview. Steroids 2003; 68:981-93.

3. Evans RM. The steroid and thyroid hormone receptor super-family. Science 1988;240:889-95.

4. Fernández-Valdivia R, Mukherjee A, Mulac-Jericevic B, Conneely OM, DeMayo FJ, Amato P, et al. Revealing progesterone's role in uterine and mammary gland biology: insights from the mouse. Semin Reprod Med 2005;23:22-37.

5. Spitz I. Clinical applications of progesterone receptor antagonists and selective progesterone receptor modulators. Endocrinologist 2005; 15:391400.

6. Eisinger SH, Meldrum S, Fiscella K, le Roux HD, Guzick DS. Low-dose mifepristone for uterine leiomyomata. Obstet Gynecol 2003;101:243-50.

7. Baird DT, Brown A, Critchley HO, Williams AR, Lin S, Cheng L. Effect of long-term treatment with lowdose mifepristone on the endometrium. Hum Reprod 2003;18:61-8.

8. Bray J, Zhang Z, Winneker RC, Lyttle CR. Regulation of gene expression by PRA-910, a novel progesterone receptor modulator, in T47D cells. Steroids 2003; 68:995-1003.

9. Blithe D, Blithe DL, Nieman LK, Blye RP, Stratton P, Passaro M. Development of the selective progesterone receptor modulator CDB-2914 for clinical indications. Steroids 2003;68:1013-7.

10. Chwalisz K, Mattia-Goldberg K, Lee M, Elger W, Edmonds A. Treatment of endometriosis with the novel selective progesterone receptor modulator (SPRM) asoprisnil. Fertil Steril 2004;82:S83S84.

11. Creinin MD, Schreiber CA, Bednarek P, Lintu H, Wagner MS, Meyn LA. Mifepristone and misoprostol administred simultaneosly versus 24 hours apart 
for abortion: a randomized controlled trial. Obstet Gynecol 2007;109:885-94.

12. Guest J, Chien PF, Thomson MA, Kosseim ML. Randomised controlled trial comparing the efficacy of same-day administration of mifepristone and misoprostol for termination of pregnancy with the standard 36 to 48 hour protocol. BJOG 2007;114:207-15.

13. Schreiber C, Creinin M. Mifepristone in abortion care. Semin Reprod Med 2005;23:82-91.

14. Bracken H, Ngoc NT, Schaff E, Coyaji K, Ambardekar S, Westheimer E, et al. Mifepristone followed in 24 hours to 48 hours by misoprostol for late first-trimester abortion. Obstet Gynecol 2007;109:895-901.

15. Fisher M, Bhatnagar J, Guarner J, Reagan S, Hacker JK, Van Meter SH, et al. Fatal toxic shock syndrome associated with Clostridium sordellii after medical abortion. N Engl J Med 2005;353:2352-60.

16. Bartley J, Baird DT. A randomised study of misoprostol and gemeprost in combination with mifepristone for induction of abortion in the second trimester of pregnancy. BJOG 2002;109:1290-4.

17. Wagaarachchi PT, Ashok PW, Narvekar NN, Smith NC, Templeton A. Medical management of late intrauterine death using a combination of mifepristone and misoprostol. BJOG 2002;109:443-7.

18. Neilson JP. Mifepristone for induction of labour. Cochrane Database of Systematic Reviews 2000, Issue 4. Art. No.: CD002865.

19. Wing DA, Fassett MJ, Mishell DR. Mifepristone for preinduction cervical ripening beyond 41 weeks gestation: a randomized controlled trial. Obstet Gynecol 2000;96:543-8.

20. Hapangama DK, Brown A, Glasier A, Baird DT. Feasibility of administering mifepristone as a once a month contraceptive pill. Hum Reprod 2001;16:1145-50.

21. Verbost PM, Hanssen RG, Korver GH, Mulders TM. ORG 33628 and ORG 31710 to control vaginal bleeding in progestine-only contraceptive regimens. Semin Reprod Med 2005;23:101-11.

22. von Hertzen H, Van Look PF. Antiprogestine for contraception? Semin Reprod Med 2005; 23:92-100.

23. Hegele-Hartung C, Mootz U, Beier HM. Luteal control of endometrial receptivity and its modification by progesterone antagonists. Endocrinology 1992; 131:2446-60.
24. Han S, Sidell N. RU486-induced growth inhibition of human endometrial cells involves the nuclear factorkappa B signaling pathway. J Clin Endocrinol Metab 2003;88:713-9.

25. Kettel LM, Murphy AA, Morales AJ, Yen SS. Preliminary report on the treatment of endometriosis with low-dose mifepristone (RU 486). Am J Obstet Gynecol 1998;178:1151-6.

26. Kim JJ, Wang J, Bambra C, Das SK, Dey SK, Fazleabas AT. Expression of cyclooxygenase-1 and -2 in the baboon endometrium during the menstrual cycle and pregnancy. Endocrinology 1999;140:2672-8.

27. Chwalisz K, Pérez MC, DeManno D, Winkel C, Schubert G, Elger W. Selective progesterone receptor modulator development and use in the treatment of leiomyomata and endometriosis. Endocrine Reviews 2006;26:423-38.

28. Chwalisz K, Elger W, Stickler T, Mattia-Goldberg C, Larsen L. The effects of 1-month administration of asoprisnil (J867), a selective progesterone receptor modulator in healthy premenopausal women. Hum Reprod 2005;20:1090-9.

29. Greb RR, Heikinheimo O, Williams RF, Hodgen GD, Goodman AL. Vascular endothelial growth factor in primate endometrium is regulated by oestrogenreceptor and progesterone-receptor ligands in vivo. Hum Reprod 1997;12:1280-92.

30. Steinauer J, Pritts EA, Jackson R, Jacoby AF. Systematic review of mifepristone for the treatment of uterine leiomyomata. Obstet Gynecol 2004;103:1331-6.

31. Zeng C, Gu M, Huang H. A clinical control study on the treatment of uterine leiomyoma with gonadotrophin releasing hormone agonist or mifepristone. Zhonghua Fu Chan Ke Za Zhi 1998;33:490-2.

32. Chwalisz K, DeManno D, Garg R, Larsen L, MattiaGoldberg C, Stickler T. Therapeutic potential for the selective progesterone receptor modulator asoprisnil in the treatment of leiomyomata. Semin Reprod Med 2004;22:113-9.

33. Goyeneche A, Caron R, Telleria C. Mifepristone inhibits ovarian cancer cell growth in vitro and in vivo. Clin Cancer Res 2007;13:3370-9.

34. Koivisto-Korander R, Leminen A, Heikinheimo O. Mifepristone as treatment of recurrent progesterone receptor-positive uterine leiomyosarcoma. Obstet Gynecol 2007;109:512-4. 